\title{
ANÁLISE DE TEXTURA CRISTALOGRÁFICA POR DIFRAÇÃO DE RAIOS X EM AÇOS IF COM DIFERENTES ADIÇÕES DE TITÂNIO, NIÓBIO E FÓSFORO*
}

\author{
Andrea Pedroza da Rocha Santos ${ }^{1}$ \\ Adriana da Cunha Rocha ${ }^{2}$ \\ Leonardo Sales Araújo
}

\section{Resumo}

Os aços IF (do inglês "interstitial free") encontram ampla aplicação na indústria automobilística devido à sua superior estampabilidade. A textura cristalográfica desenvolvida durante a laminação de aços IF tem um papel fundamental na conformabilidade das chapas laminadas, sendo desejável o desenvolvimento de uma forte fibra gama e fraca fibra alfa. Neste trabalho, três tipos de aços IF, com diferentes adições de titânio, nióbio e fósforo, foram submetidos a análise de textura cristalográfica por difração de raios $X$, de modo a avaliar o impacto dos tratamentos termomecânicos realizados e da variação de composição química nas orientações cristalográficas formadas. As texturas foram analisadas utilizando-se funções de distribuição de orientação (ODF). Os resultados das seções de ODF foram comparados a valores anteriormente obtidos para os coeficientes de anisotropia normal e planar destes mesmos aços. A textura nas amostras laminadas de fato apresentou fibra gama intensificada e fibra alfa parcial. O aço IF-TiNbP foi o que apresentou melhor textura para estampagem profunda. No entanto, foi observada componente Goss na superfície do mesmo.

Palavras-chave: Textura Cristalográfica; Aços IF; Difração de raios X.

\section{TEXTURE ANALYSIS BY X-RAY DIFFRACTION OF IF-STEELS WITH DIFFERENT ADDITIONS OF TITANIUM, NIOBIUM AND PHOSPHORUS}

\begin{abstract}
Interstitial free steels (IF steels) find wide application in the automotive industry, due to their excellent drawability. The crystallographic texture developed during the rolling of IF steels plays a fundamental role in the formability of the rolled sheets, being desirable the development of a strong gama fiber and weak alfa fiber. In this work, three types of IF steels with different additions of titanium, niobium and phosphorus were subjected to crystallographic texture analysis by $x$-ray diffraction, in order to evaluate the impact of the thermomechanical treatments performed and the variation of chemical composition in the crystallographic orientations formed. The textures were analyzed using orientation distribution functions (ODF). The results of the ODF sections were compared to previously obtained values for the normal and planar anisotropy coefficients of these same steels. The texture in the rolled samples did indeed show increased gama fiber and partial alfa fiber. The IF-TiNbP steel presented the best texture for deep drawing. However, Goss component was observed on the surface of this same steel.
\end{abstract}

Keywords: Crystallographic texture; IF steels; X-ray diffraction.

1 Engenheira Metalúrgica, Mestrado em Engenharia Metalúrgica e de Materiais pela COPPE/UFRJ, Aluna de Doutorado do Programa de Pós Graduação de Engenharia Metalúrgica e de Materiais (PEMM) da COPPE/UFRJ, Rio de Janeiro, RJ, Brasil.

2 Engenheira Metalúrgica e de Materiais, Doutora em Engenharia Metalúrgica e de Materiais, Professora Adjunta do Programa de Engenharia Metalúrgica e de Materiais (PEMM) da COPPE/UFRJ, Rio de Janeiro, RJ, Brasil

3 Graduação em Engenharia Metalúrgica, Mestrado em Engenharia de Produção, Doutorado em Engenharia Metalúrgica e de Materiais, Professor Adjunto do Programa de Engenharia Metalúrgica e de Materiais (PEMM) da COPPE/UFRJ, Rio de Janeiro, RJ, Brasil. 


\section{INTRODUÇÃO}

Aços livres de intersticiais, também conhecidos como aços IF (do inglês "interstitial free"), são assim chamados devido ao controle para minimizar elementos intersticiais na matriz, resultando em um aço altamente dúctil. Eles apresentam matriz ferrítica, com estrutura CCC, e uma composição com ultrabaixo teor de carbono e nitrogênio (inferior a 0,003\%peso).

Os aços IF foram inicialmente desenvolvidos durante a década de 1970, com o objetivo principal de se produzirem aços com maior conformabilidade para aplicações em estampagem profunda. Eles começaram a ser produzidos em grande escala quando passaram a ser requisitados pela indústria automobilística moderna, sendo utilizados principalmente na confecção de painéis automotivos [1].

Nestes aços, os elementos intersticiais são mantidos em teores muito baixos devido à utilização de modernas técnicas de desgaseificação a vácuo $(R H)$ e em virtude da adição de elementos microligantes como titânio e/ou nióbio. A adição desses elementos leva à formação de precipitados de carbetos e nitretos, de modo que a matriz ferrítica do aço IF fique praticamente livre de carbono e nitrogênio.

Geralmente, os aços IF são distinguidos pelo elemento que estabiliza os solutos insterticiais, sendo principalmente classificados em: estabilizados por titânio (IF-Ti) [2], por nióbio (IF-Nb) [3] e estabilizados juntamente por titânio e nióbio (IF-TiNb) [4]. Com o objetivo de se obter um aço de melhor resistência mecânica, podem ainda existir aços IF com adição de outros elementos de liga, tais como fósforo, silício e manganês, promovendo assim o endurecimento por solução sólida. Esse tipo de aço é comumente conhecido como aço IF de alta resistência (IF-HS).

Ultimamente, os produtores de aço têm procurado reduzir o custo de produção ao mesmo tempo em que buscam aumentar o desempenho dos produtos. Além disso, a minimização do impacto ambiental é de importância crescente nos últimos tempos. Soluções sustentáveis para estes desafios podem ser conseguidas somente por meio da pesquisa e do aperfeiçoamento contínuos em todas as fases do processo de produção dos aços.

Nos aços IF, a maior conformabilidade adquirida é acompanhada de perda na resistência mecânica, o que se tornou o grande desafio no desenvolvimento desses aços [5]. Desta forma, são necessários cada vez mais estudos a fim de se entender como os parâmetros de processos e a variação de composição química afetam as propriedades do aço, e, consequentemente, seu desempenho em estampagem.

O presente estudo tem o objetivo de complementar os ensaios já realizados em três tipos de aços IF com diferentes adições de titânio, nióbio e fósforo, contribuindo assim para a escolha de melhores parâmetros de processamento e de composição química do aço, a fim de se obterem propriedades mecânicas finais de qualidade superior.

Aços IF-Ti, IF-TiNb e IF-TiNbP foram submetidos a análise de textura cristalográfica por difração de raios $\mathrm{X}$, de modo a se avaliar e comparar a textura obtida, após o processo de laminação realizado em laboratório, em função da variação de composição química.

Figuras de ODF (do inglês "Orientation Distribution Function") foram calculadas e comparadas a resultados anteriormente obtidos para os coeficientes de anisotropia normal $(\mathrm{rm})$ e planar $(\Delta \mathrm{r})$ destes mesmos aços. A textura nas amostras laminadas apresentou fibra gama intensificada e fibra alfa parcial. O aço IF-TiNbP foi o que apresentou melhor textura para aplicações em estampagem profunda. No entanto, foi observada componente Goss na superfície do mesmo. 


\section{MATERIAIS E MÉTODOS}

\subsection{Materiais}

Para a realização deste estudo foram utilizadas amostras de três diferentes tipos de aços IF: IF-Ti ou estabilizado ao titânio; IF-TiNb, estabilizado ao titânio e nióbio; e IF-TiNbP, estabilizado ao titânio e nióbio com adição de fósforo. Tal material foi estudado em outros trabalhos de pesquisa [6,7]. A composição química de cada um destes aços é apresentada na Tabela 1.

Tabela 1 - Composição química (\%peso) dos aços IF utilizados.

\begin{tabular}{cccccccccc}
\hline Aço IF & $\mathbf{C}$ & $\mathbf{N}$ & $\mathbf{N b}$ & $\mathbf{T i}$ & $\mathbf{P}$ & $\mathbf{A l}$ & $\mathbf{S}$ & $\mathbf{M n}$ & $\mathbf{S i}$ \\
$\mathbf{T i}$ & 0,0021 & 0,0025 & 0,0018 & 0,0820 & 0,0099 & 0,0346 & 0,0063 & 0,1250 & 0,0180 \\
\hline TiNb & 0,0022 & 0,0038 & 0,0160 & 0,0700 & 0,0083 & 0,0361 & 0,0093 & 0,1120 & 0,0710 \\
\hline TiNbP & 0,0022 & 0,0037 & 0,0288 & 0,0334 & 0,0298 & 0,0384 & 0,0071 & 0,1750 & 0,0500 \\
\hline
\end{tabular}

Os aços foram cedidos pela ThyssenKrupp CSA, cada amostra tendo sido retirada da parte central das placas lingotadas para evitar contaminação de corridas anteriores e reduzir possíveis variações de processo. Foram feitos cortes em blocos, apresentando dimensões aproximadas de $80 \times 50 \times 25 \mathrm{~mm}$.

As amostras passaram por um processamento em laboratório da UFRJ, que incluiu as etapas de reaquecimento, laminação a quente, laminação a frio e recozimento, obtendo-se no final chapas laminadas com $1 \mathrm{~mm}$ de espessura.

\subsection{Textura por difração de raios $X$}

Uma vez que existe uma forte relação entre a orientação preferencial dos grãos e a formabilidade da chapa, o controle da textura cristalográfica é essencial para otimizar a estampabilidade dos aços [8].

Nos aços livres de intersticiais (IF), muito utilizados na indústria automobilística, uma combinação adequada de laminação e recozimento resulta em chapas com texturas que apresentam suas orientações principais ideais localizadas em duas fibras: fibra a (alfa) e fibra $\gamma$ (gama) [9].

A fibra alfa é também chamada de fibra DL, pois inclui todas as orientações com direção <110> paralela à direção de laminação. Ela é considerada prejudicial para requisitos de estampagem profunda.

A fibra gama, também conhecida por fibra DN, é caracterizada por apresentar grãos orientados preferencialmente com seu plano $\{111\}$ paralelo à superfície da chapa, ou seja, com as normais aos planos $\{111\}$ paralelas à direção normal, sendo também conhecida por fibra $\{111\}$. Essa fibra é considerada benéfica para operações de estampagem profunda.

Há ainda a fibra $\varepsilon$, também conhecida por fibra DT, composta por componentes de cisalhamento provenientes de deformação não homogênea. Suas principais componentes são o cubo girado $\{001\}<110>$ e a orientação Goss $\{011\}<100>$. Ambas são consideradas prejudiciais às propriedades de estampagem e devem ser evitadas.

Para os ensaios de textura cristalográfica, as amostras após o processamento foram cortadas em pequenos corpos de prova com dimensões de 20 × $20 \mathrm{~mm}$. Foram preparados corpos de provas tanto da superfície quanto da meia espessura da chapa laminada, a fim de se analisarem os resultados de textura após a laminação de uma forma completa, observando-se a qualidade tanto superficial como de centro de espessura propícia à conformação mecânica. 
Todos os corpos de prova foram lixados com lixas de granulometrias 80 a 1500 mesh. Em seguida, foi feito um polimento mecânico com pasta de diamante de $1 \mu \mathrm{m}$ e um ataque químico com uma solução de Nital 5\% para remover o efeito de superfície.

O equipamento utilizado para as análises da textura cristalográfica foi um difratômetro de raios $X$ fabricado pela empresa holandesa PANalytical, disponível no Laboratório de Raios $X$ do Instituto Militar de Engenharia (IME). A fonte de radiação utilizada foi de cobalto, cujo comprimento de onda é igual a 1,789Å. A tensão e a corrente de operação foram ajustadas em $45 \mathrm{~mA}$ e $40 \mathrm{KV}$, e o ensaio foi feito em foco ponto (point focus).

Em todas as amostras foram coletados os resultados de difração relativos aos planos (110), (200) e (211), característicos dos materiais com estrutura cristalina cúbica de corpo centrado (CCC).

O resultado da análise de textura cristalográfica foi ilustrado por meio das funções de distribuição de orientações (ODF's), em que se podem observar as intensidades dos componentes de textura em função dos ângulos de rotação (Euler). As figuras de ODF's foram geradas pelo programa popLA (preferred orientation package - Los Alamos).

Serão utilizadas notações de Roe [10], e apenas a seção $\phi=45^{\circ}$ será estudada, uma vez que a mesma contém todas as orientações principais apresentadas em chapas de aço IF destinadas à estampagem profunda.

\section{RESULTADOS E DISCUSSÃO}

Os resultados dos ensaios de textura cristalográfica por difração de raios $X$ estão apresentados por meio de funções de distribuição de orientação, Figura 1. 
a)

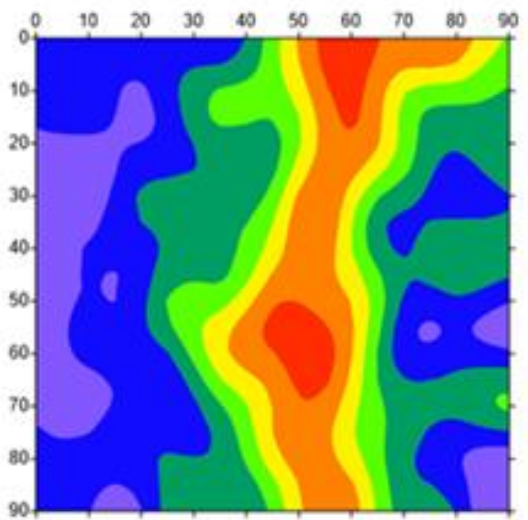

c)

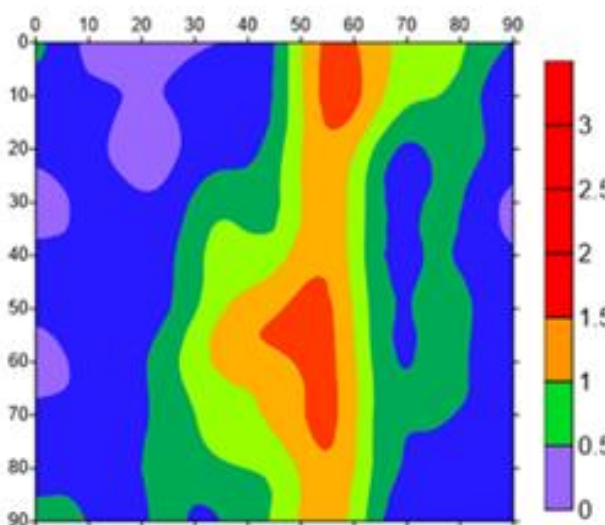

e)

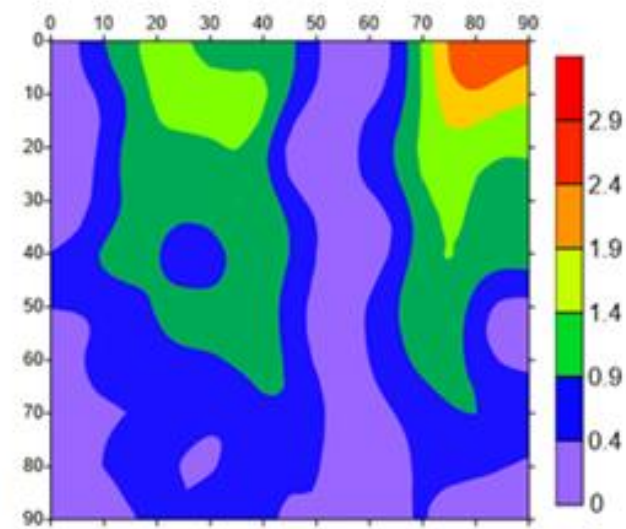

b)

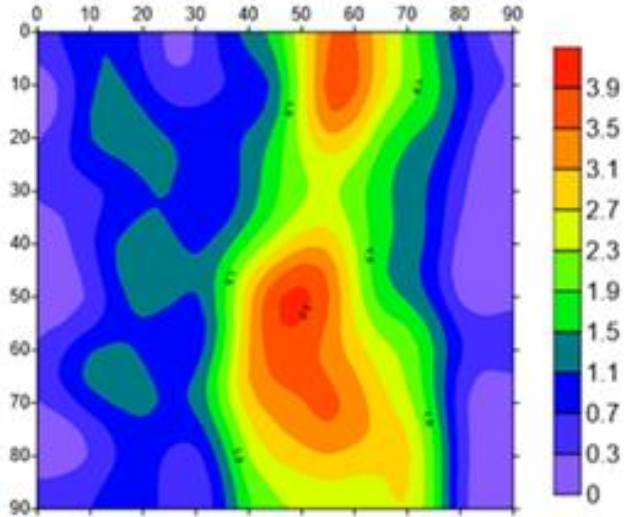

d) 190

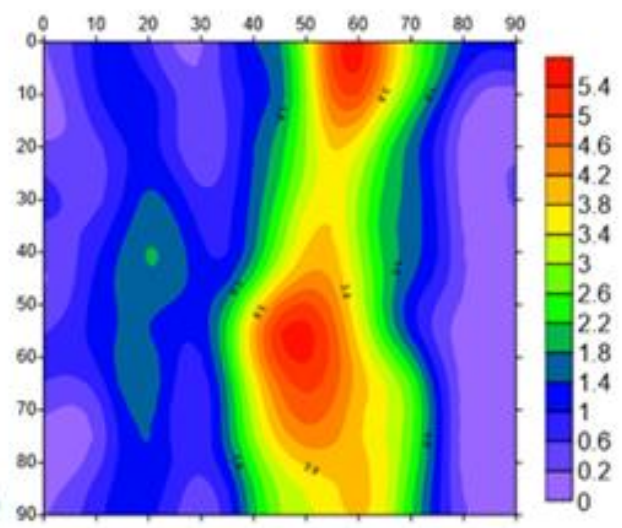

f)

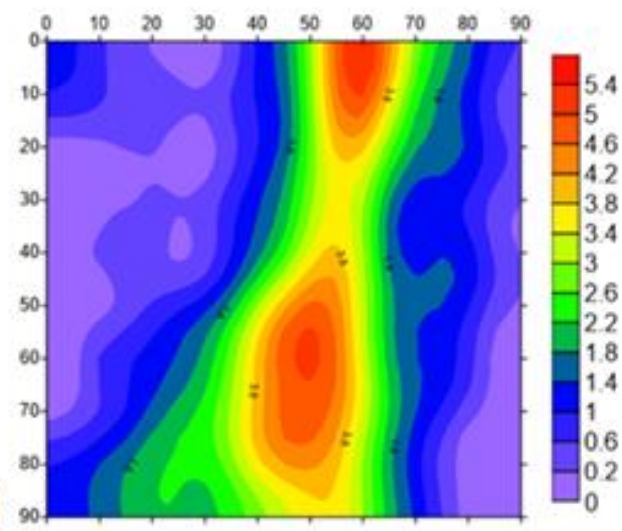

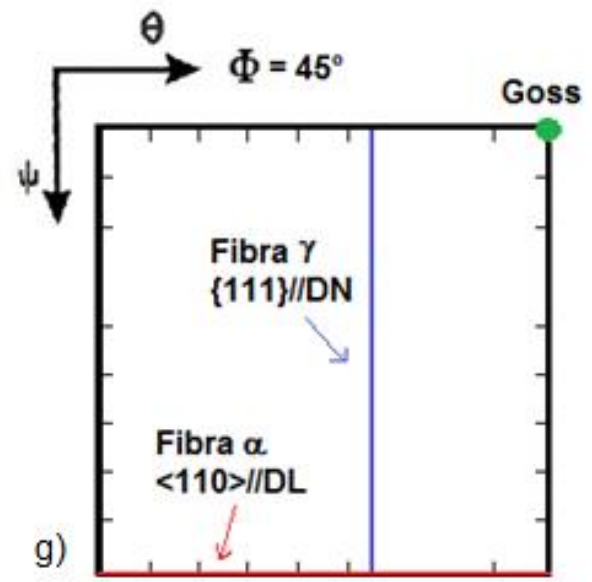


Figura 1 - ODF's das amostras: a) medições na superfície da chapa laminada - Aço IF-Ti, b) medições na meia espessura da chapa laminada - Aço IF-Ti, c) medições na superfície da chapa

laminada - Aço IF-TiNb, d) medições na meia espessura da chapa laminada - Aço IF-TiNb, e) medições na superfície da chapa laminada - Aço IF-TiNbP, f) medições na meia espessura da chapa laminada - Aço IF-TiNbP, g) ábaco para $\phi=45^{\circ}$, notação de Roe.

Conforme pode ser observado na Figura 1, em quase todos os casos, após a laminação, o material dos aços IF-Ti, IF-TiNb e IF-TiNbP passam a apresentar textura de fibra gama. Somente no caso da figura 1e, na superfície da amostra do aço IFTiNbP, a fibra gama é totalmente ausente, apresentando a ODF maior intensidade na região da componente Goss.

É conhecido que variadas componentes de textura influenciam nos diferentes valores de $r_{m}$ e $\Delta r$. Ao compararmos os resultados da análise de textura do presente trabalho com os resultados dos coeficientes de anisotropia obtidos no trabalho de MOTA [6], tabela 2, observamos algumas características distintas para cada aço IF analisado.

\begin{tabular}{ccccccc} 
Aço IF & $\mathbf{r}_{\mathbf{m}}$ & $\mathbf{\Delta r}$ & $\mathbf{n}$ & $\begin{array}{c}\text { Limite de } \\
\text { Escoamento } \\
\text { (MPa) }\end{array}$ & $\begin{array}{c}\text { Tabela } 2-\text { Resultados de ensaios de tração uniaxial [6]. } \\
\text { (MPa) }\end{array}$ & $\begin{array}{c}\text { Alongamento } \\
\text { Total }\end{array}$ \\
\hline Ti & 1,92 & 1,98 & 0,296 & 105,19 & 260,21 & 0,41 \\
\hline TiNb & 1,16 & 1,11 & 0,304 & 120,84 & 278,40 & 0,42 \\
\hline TiNbP & 1,28 & $-0,09$ & 0,299 & 162,09 & 322,54 & 0,34 \\
\hline
\end{tabular}

\subsection{Aços IF-Ti}

No caso dos aços IF com adições de titânio (IF-Ti), MOTA [6] obteve o valor de $r_{m}=1,92$. Tal fato foi confirmado pela presença de fibra gama na chapa laminada tanto na superfície como na meia espessura, indicando uma forte presença desta fibra na peça como um todo.

Ao analisarmos o resultado para o coeficiente de anisotropia planar $(\Delta r=1,98)$, entretanto, verifica-se que este apresenta um valor muito diferente de 0 , que seria 0 valor desejado para garantir uma boa estampabilidade e evitar a formação de orelhas no produto final. Uma das razões para este valor pode ser vista pelos resultados obtidos para a componente Goss. Um comportamento de textura ideal para a aplicação em estampagem profunda inclui uma ausência ou baixa intensidade da componente Goss no material [11]. De fato, como apresentado pelos trabalhos de DANIEL [12] e LEQUEU [13], a componente Goss é caracterizada por apresentar uma forte tendência de contribuição para elevados valores de coeficiente de anisotropia normal e planar.

\subsection{Aços IF-TiNb}

Para os aços IF com adições de titânio e nióbio (IF-TiNb), nota-se que, na meia espessura, a fibra gama se encontra de forma forte, apresentando elevada média de intensidade.

Analisando-se agora os resultados obtidos para $r_{m}$ e $\Delta r$, verifica-se que, em comparação aos aços IF-Ti, os valores absolutos destes coeficientes diminuíram. É importante observar que a fibra gama obtida na superfície da chapa laminada do aço IF-TiNb apresenta valores similares em toda a sua extensão, estando presente de forma mais homogênea no material. Junto a isso, sabe-se que a fibra gama melhora 
a formabilidade (pela promoção de valores razoáveis de $r_{m}$ ) e diminui a influência do coeficiente de anisotropia planar no material, principalmente pela contribuição da componente $\{111\}<112>$, que, neste caso, apresentou valor máximo de 5,1 $[14,15]$. Sendo assim, este pode ser o motivo pelo qual o valor de $r_{m}$ manteve-se acima de 1,0 e o valor de $\Delta r$ diminuiu. Ainda assim, o valor do coeficiente planar continua elevado em relação ao ideal e, mais uma vez, pode-se associá-lo aos valores da componente Goss obtidos, pois a mesma está presente no material, mas de forma mais fraca quando comparada ao aço IF-Ti.

\subsection{Aços IF-TiNbP}

Os aços IF com adições de titânio, nióbio e fósforo (IF-TiNbP) apresentaram resultados interessantes ao longo da espessura. Observa-se claramente pelas ODF's que os processos de laminação anularam completamente a fibra gama na superfície da chapa laminada. Entretanto, essa fibra foi fortemente favorecida na meia espessura.

No que diz respeito à componente Goss, na meia espessura esta encontra-se presente de forma bem fraca. Na superfície, por sua vez, a mesma apresenta o valor mais elevado encontrado em todos os aços, atingindo um nível de 2,5. Este resultado compromete as propriedades de estampabilidade, principalmente porque, como mencionado anteriormente, a fibra gama foi totalmente suprimida nesta mesma região. É interessante notar que, apesar do resultado insatisfatório na superfície do aço IF-TiNbP, os valores das fibras à meia espessura são considerados bons em termos de estampabilidade, i.e., baixo valor de Goss, alto valor de fibra gama e reduzido valor de fibra alfa.

Analisando os resultados obtidos por MOTA [6] para os coeficientes de anisotropia normal e planar, evidencia-se também que estes apresentaram a melhor combinação de valores dentre os três aços, isto é, $r_{m}>1$ com $\Delta r \approx 0$, apesar de apresentar uma anisotropia normal abaixo de 2,0, valor esperado para aços IF [16].

Ainda assim, é preciso levar em conta o efeito ocorrido na superfície da chapa laminada, que leva à supressão de fibra gama e ao aumento da orientação Goss.

$A$ adição de fósforo aos aços IF tem como principal objetivo promover o endurecimento por solução sólida, aumentando assim sua resistência. De fato, o aço IF-TiNbP foi o que apresentou o maior limite de resistência (322,54 MPa). Entretanto, uma maior resistência do material pode levar a um maior atrito entre a superfície da chapa e os cilindros de laminação. Esse atrito ocasiona a geração de tensões de cisalhamento, que levam à nucleação de orientações preferenciais nas direções da componente Goss. Este fato foi observado nos trabalhos de LEE [17] e BRUNA [18], que analisaram o efeito da interação de cilindros com lubrificação insuficiente na formação de componentes de cisalhamento.

A adição de fósforo também promove a formação de precipitados do tipo FeTiP. Estes precipitados, no entanto, consomem o excesso de titânio - que formaria carbetos - no aço IF, deixando solutos de carbono presentes na matriz [19]. Com efeito, o trabalho de SEGUNDO [7], neste mesmo material, evidenciou a presença de precipitados de FeTiP.

É importante ressaltar que a presença de carbono em solução, por sua vez, está relacionada com a ocorrência de descontinuidades no limite de escoamento [20]. MOTA [6] observou tal fato em seu trabalho, em que o aço IF-TiNbP apresentou limite de escoamento descontínuo durante carregamento. A combinação de todos estes fatores leva à indicação de que a presença de uma forte componente Goss na superfície está relacionada com o limite de escoamento descontínuo. 
Esta interpretação é suportada pelo fato de que uma elevada quantidade de carbono em solução está associada a uma diminuição do coeficiente de anisotropia normal $\left(r_{m}\right)$ devido à nucleação de componentes de textura desfavoráveis, ou seja, diminuição da intensidade relativa da fibra \{111\} [20]. Com efeito, HÖLSCHER [21] observou que a elevação do teor de carbono na matriz aumenta a formação de orientação Goss, enquanto no caso do carbono precipitado, a densidade de orientação Goss é fortemente reduzida. A razão para esse fato é que o carbono em solução leva a mais deformação não homogênea, formando bandas de cisalhamento.

\subsection{Considerações finais}

Comparando os resultados das orientações cristalográficas formadas nos três aços, observa-se que o aço IF-TiNbP apresenta, na sua meia espessura, o resultado mais satisfatório em termos de formação de fibras gama, alfa e orientação Goss. Os valores obtidos atendem de forma plena às condições de otimização de textura cristalográfica para melhor aplicação em processos de estampagem profunda.

Os efeitos observados na superfície deste aço, como mencionado, surgiram provavelmente por uma combinação de aumento de resistência com a presença de precipitados FeTiP na matriz ferrítica, ambos promovendo a formação de componente de cisalhamento (Goss). Especial atenção deve ser dada então a dois pontos principais. O primeiro é relacionado à lubrificação utilizada durante o processo de laminação, que, se empregada de forma insuficiente, pode levar à supressão de fibra gama e ao aumento de orientação Goss. O segundo refere-se à formação de precipitados de FeTiP, que deve ser mitigada ao máximo para evitar a presença de carbono em solução, já que esta condição leva a descontinuidades no limite de escoamento do material e também ao aumento da orientação Goss.

Outra observação a ser feita seria quanto aos valores obtidos para anisotropia normal dos aços IF-TiNb e IF-TiNbP. Ambos apresentaram valores inferiores ao esperado para aços IF, que geralmente encontram-se próximos a 2,0. Tal fato pode estar relacionado aos parâmetros de processo utilizados. Provavelmente, a sequência de processamento realizada não é adequada para os três tipos de aços, principalmente para o aço IF-TiNbP, que além de apresentar valor de anisotropia normal abaixo do esperado, apresentou limite de escoamento descontínuo.

\section{CONCLUSÃO}

- Foi possível relacionar os valores dos coeficientes de anisotropia planar e normal dos três aços analisados com os valores obtidos para as fibras gama, alfa e componente Goss;

- Comparando-se os três aços, o aço IF-TiNbP apresentou um melhor comportamento em relação à textura, sendo necessário apenas fazer uma ressalva para a superfície da chapa laminada;

- Os resultados obtidos na análise de textura indicam claras diferenças entre o comportamento da superfície da chapa laminada e de sua meia espessura, apresentando a fibra gama valores mais baixos na superfície. Este fato indica que deve ser dada especial atenção à lubrificação durante o processo de laminação;

- O aço IF-TiNbP foi o que apresentou a superfície da chapa laminada mais afetada em termos de textura, com ausência de fibra gama e forte presença da componente Goss (110)[001]. Além disso, o mesmo foi o único que apresentou limite de escoamento descontínuo. Ambos os fatos indicam que o carbono pode ter permanecido em solução sólida; 
- A mesma sequência de processamento provavelmente não é adequada para os três tipos de aços, principalmente para o aço IF-TiNbP, que apresentou valor de anisotropia normal abaixo do esperado e limite de escoamento descontínuo.

\section{REFERÊNCIAS}

1 ITAMI, A., et al., "Development of New Formable Cold-Rolled Sheet Steels for Automobile Body Panels", Nippon Steel Technical Report, v.64, pp. 26-32, January 1995.

2 SHI, J., WANG, X., "Comparison of Precipitate Behaviors in Ultra-Low Carbon, TitaniumStabilized Interstitial Free Steel Sheets under Different Annealing Processes", Journal of Materials Engineering and Performance, v. 8, n.6, pp. 641-648, 1999.

3 HOOK, R.E., HECKLER, A.J., ELIAS, J.A., "Texture in Deep-Drawing Columbium (Nb) Treated Interstitial-Free Steels", Metallurgical and Materials Transactions A, v.6, n.9, pp. 1683-1692, September 1975.

4 TOKUNAGA, Y., YAMADA, M., ITOH, K., "Effect of Combined Addition of $\mathrm{Nb}$ and Ti on the Mechanical Properties of Extra Low Carbon Steel Sheets", ISIJ International, v.73, n.2, pp. 341-348, 1987.

5 TAKECHI, H., "Metallurgical Aspects on Interstitial Free Sheet Steel from Industrial Viewpoints", ISIJ International, v.34, n.1, pp. 1-8, 1994.

6 MOTA, T.C., 2015, Análise da anisotropia de propriedades mecânicas de aços IF. Projeto de Graduação, UFRJ, Rio de Janeiro, RJ, Brasil.

7 SEGUNDO, H.V., 2016, Efeito da adição de $\mathrm{P}$, Ti e Nb na evolução da microestrutura dos aços IF. Dissertação de M.Sc., COPPE-UFRJ, Rio de Janeiro, RJ, Brasil.

8 BUNGE, H.J., Texture Analysis in Materials Science. Elsevier, 1969.

9 ENGLER, O., RANDLE, V., Introduction to texture analysis: macrotexture, microtexture and orientation mapping. Boca Raton, Taylor \& Francis Group, 2010.

10 ROE, R.J., "Description of crystallite orientation in polycrystalline materials. III. General solution to pole figure inversion", Journal of Applied Physics, v.36, n.6, pp. 2024-2031, June 1965.

11 TOMITZ, A., KASPAR, R. "Deep-Drawing quality of cold rolled sheet made of ferritically rolled hot strip", Steel Research, v.71, n.12, pp. 504-510, December 2000.

12 DANIEL, D., JONAS, J.J., "Measurement and prediction of plastic anisotropy in deepdrawing steels" Metallurgical Transactions A, v.21, n.1, pp. 331-343, January 1990.

13 LEQUEU, PH., JONAS, J.J., "Modeling of the plastic anisotropy of textured sheet" Metallurgical and Materials Transactions A, v.19, n.1, pp. 105-120, January 1988.

14 LEWIS, D.B., PICKERING, F.B. "Development of recrystallization textures in ferritic stainless steels and their relationship to formability", Metals Technology, v.10, n.1, pp. 264-273, 1983.

15 RAABE, D., LÜCKE, K. "Influence of particles on recrystallization textures of ferritic Stainless Steels", Steel Research, v.63, n.10, p. 457-467, 1992.

16 WANG, Z., WANG, X., "A new technology to improve the $\overline{\mathrm{r}}$-value of interstitial-free (IF) steel sheet", Journal of Materials Processing Technology, v.113, pp. 659-661, June 2001.

17 LEE, S.H., LEE, D.N., "Shear rolling and recrystallization textures of interstitial-free steel sheet", Materials Science and Engineering, v.249, n.1, pp 84-90, June 1998.

18 BRUNA, R.G., "Effects of hot and warm rolling on microstructure, texture and properties of low carbon steel", Rem: Revista Escola de Minas, v.64, n.1, pp. 57-62, Março 2011.

19 JIA, J., ZHU, W., SONG, X., "Precipitation Behavior of FeTiP in Ti-added Interstitial Free High Strength Steels", Journal of Iron and Steel Research, v.23, n.7, pp. 692-698, July 2016.

20 HUTCHINSON, W.B., "Development and Control of Annealing Textures in Low-Carbon Steels", International Metals Reviews, v.29, n.1, pp. 25-42, 1984.

21 HÖLSCHER, M., RAABE, D., LÜCKE, K., "Rolling and recrystallization textures of bcc steels", Steel Research, v.62, n.12, pp. 567-575, December 1991. 